\title{
nature
}

cell biology

\section{Signalling and adhesion take centre stage}

In this special issue we

have brought together an

exciting collection of

commissioned pieces in

the field of signalling and

adhesion from world-

renowned experts...
$\mathbf{N}$ ature Cell Biology is pleased to welcome all its readers to this special 'Focus on Signalling and Adhesion' issue. We hope this issue will appeal not only to experts in the field of signalling and adhesion, but to all our readers.

The fields of signalling and adhesion research were once separate and distinct from one another. Even within these fields, researchers would often define themselves as integrin researchers, cadherin experts or epidermal growth factor receptor (EGFR) signalling specialists. Over the last 20 years an enormous amount of research has challenged the way we view these fields. Instead of visualizing individual adhesion receptors or signalling pathways that activate a linear downstream signalling cascade, we now know that adhesion molecules and growth factor receptors are involved in an intimate 'crosstalk' that regulates a myriad of downstream signalling events. Individual adhesion molecules, for example the epithelial adhesion molecule E-cadherin, have been shown to localize and interact with growth factor signalling receptors, such as the EGFR. This not only introduces complex interactions between the different pathways but leads to a network of interacting molecules that can then regulate the cellular response generated by the external signals the cell receives. Nowhere is this crosstalk more apparent than in the field of integrin research.

In this special issue we have brought together an exciting collection of commissioned pieces in the field of signalling and adhesion from world-renowned experts, complemented by primary research pieces in this area. To ensure all cell biologists with an interest in this topic can access these commissioned pieces, we have made the entire issue free online during April 2002 at http://cellbio.nature.com.

Furthermore, we are launching a new 'Focus on Signalling and Adhesion' site on our homepage that will contain all the pieces we have previously published in this field. This site will complement our existing focus sites and will be continuously updated on a monthly basis. Please see http://cellbio.nature.com/focus/signallingadhesion for further details.

\section{Crosstalk in focus}

In this special issue we hope you will enjoy the primary research papers, which include an article from Gary Bokoch and colleagues (p.294), which indicates that the nucleotide exchange factor GEF-H1 mediates crosstalk between microtubules and the actin cytoskeleton.

In addition we have a selection of commissioned pieces. These include a Historical perspective from Cindy Miranti and Joan Brugge on integrins and signal transduction (p.E83). This piece is accompanied by a timeline highlighting all the key events in the field of integrin research. This timeline can be downloaded from our homepage. This historical piece is complemented by two Reviews; one by Colin Jamora and Elaine Fuchs on the regulation of adhesion by the cytoskeleton (p.E101) and a second by Donna Webb, Thomas Parsons and Rick Horwitz on cell migration (p.E97). There is also a book review, website review, commentary on integrin-signalling crosstalk from Mark Ginsberg and Martin Schwartz (p.E65), and a technology review on the latest tools used to study cell adhesion and migration from Ken Jacobson and colleagues (p.E91).

We hope all our readers enjoy this superb collection of commissioned articles and the free online access in April 2002 and we look forward to hearing your feedback. 